\title{
Nonverbal working memory of humans and monkeys: Rehearsal in the sketchpad?
}

\author{
DAVID A. WASHBURN \\ Georgia State University, Atlanta, Georgia \\ and \\ ROBERT S. ASTUR \\ University of New Mexico, Albuquerque, New Mexico
}

\begin{abstract}
Investigations of working memory tend to focus on the retention of verbal information. The present experiments were designed to characterize the active maintenance rehearsal process used in the retention of visuospatial information. Rhesus monkeys (Macaca mulatta; $N=6$ ) were tested as well as humans (total $N=90$ ) because these nonhuman primates have excellent visual working memory but, unlike humans, cannot verbally recode the stimuli to employ verbal rehearsal mechanisms. A series of experiments was conducted using a distractor-task paradigm, a directed forgetting procedure, and a dual-task paradigm. No evidence was found for an active maintenance process for either species. Rather, it appears that information is maintained in the visuospatial sketchpad without active rehearsal.
\end{abstract}

Since the inception of psychology, studies of human memory have emphasized the retention and retrieval of verbal information. Given our impressive ability to retain words in memory over short or long periods of time, as well as the ease with which words (and nonwords like nonsense syllables) can be used in recognition and recall paradigms, it is not surprising that the literature is replete with investigations of verbal learning and verbal memory. The emphasis on memory for verbal material is particularly striking in research on short-term or working memory. In fact, the capacity (or span) of working memory is typically measured by the number of words or digits that can be repeated from memory.

Equally important, albeit less investigated and understood, is the retention of nonverbal information over brief periods of time. Baddeley (1986) called this aspect of memory the visuospatial sketchpad to distinguish it from systems for retention of verbal/acoustic information, variously called the articulatory loop and the phonological store. The visuospatial sketchpad presumably is the system that (1) supports working memory for images and spatial information, (2) enables individuals to mentally rotate these images, (3) contributes to the advantage for remembering high-imagery versus low-imagery words, and (4) pro-

This research was supported in part by grants from the National Aeronautics and Space Administration (NAG2-438) and the National Institutes of Health (HD-06016), and by the College of Arts and Sciences of Georgia State University. The authors thank Duane M. Rumbaugh and the students in Introduction to Learning for their contributions to this research. Correspondence should be addressed to D. A. Washburn, Sonny Carter Life Sciences Laboratory, Department of Psychology, Georgia State University, Atlanta, GA 30303 (e-mail: Ircdaw@panther.gsu.edu).

-Accepted by previous editor, Geoffrey R. Loftus vides the basis for short-term memory in preverbal children and nonhuman animals.

Much remains unknown about the visuospatial sketchpad. Baddeley (1986) reviewed contradictory evidence as to whether its representational code is visual or spatial. Posner, Boies, Eichelman, and Taylor (1969) estimated the duration of visual working memory at around $2 \mathrm{sec}$, but Phillips and Baddeley (1971) posited that visual storage persists much longer (see also Phillips, 1983). Phillips and Christie (1977a, 1977b) reported that the span of the sketchpad is limited to one item; however, recognition studies with humans and nonhuman animals suggest that this estimate may be conservative (e.g., Sands \& Wright, 1980). In fact, Phillips and Christie (1977a) themselves reported that retention of the terminal item in a visual list was superior to that for previous items, but performance on the other items in the list was also in excess of chance. This pattern of results has recently been found for memory in monkeys as well (Washburn \& Gulledge, 1995). Finally, little is known about whether an active maintenance process operates in visuospatial memory, as is the case with rehearsal in verbal working memory.

Studies of rehearsal in the visuospatial system of humans are uncommon. It seems clear that forming an image of to-be-remembered stimuli (perhaps a form of elaborative rehearsal) results in improved recall or recognition (see, e.g., Paivio, 1971). Mentally rehearsing an action or procedure prior to executing the behavior has also been argued to improve performance in a variety of settings (see, e.g., Lejeune, Decker, \& Sanchez, 1994; cf. Zervas \& Kakkos, 1991). However, these findings do not suggest that maintenance rehearsal is required for short-term retention of visuospatial information, nor that humans utilize active rehearsal to maintain nonverbal memory. Most frequently, the existence of visual rehearsal is argued by analogy: 
Given the effectiveness and facility of verbal maintenance rehearsal, visuospatial rehearsal is assumed-as if retention implied rehearsal.

One difficulty in studying the existence of nonverbal rehearsal is the propensity for humans to translate visuospatial stimuli into information that can be rehearsed verbally (Brandimonte, Hitch, \& Bishop, 1992). When possible, humans use labeling, enumeration, and description to convert imaginal and spatial information into codes that can be rehearsed using articulatory mechanisms (an activity with implications for the existence, or at least the ineffectiveness, of visuospatial rehearsal). It seems no exaggeration to note that the biggest obstacle in the study of the visuospatial sketchpad is the confounds and artifacts that are introduced by the auditory and articulatory memory subsystems.

For this reason, research with nonhuman animals should be particularly interesting to scientists who study visual memory. The risk of marked differences in visuospatial memory between human and nonhuman species is offset by the opportunity to study visual memory in the absence of concerns for linguistic recoding. For example, rehearsal by nonhuman animals has been the specific topic of study in several experiments (reviewed by Rilling \& Neiworth, 1986; Roitblat, 1987). Four major sources of evidence have been cited to support the contention that nonhuman animals rehearse in working memory. First, recognition is impaired by performance of a distractor task during retention intervals (Kendrick \& Rilling, 1984). This finding is similar to the results of numerous experiments in which humans have poorer retention of verbal (e.g., Brown, 1958; Peterson \& Peterson, 1959) or visuospatial (Phillips \& Christie, 1977a, 1977b) information following an intervening activity.

Second, humans and other animals perform significantly more poorly on retention tests that follow a forget cue than those that follow a remember cue (Kendrick \& Rilling, 1984; Kendrick, Rilling, \& Stonebraker, 1981; Maki, 1981). Moreover, delaying the forget cue (thus allowing time for the subject to rehearse) results in systematically better probe-test performance (Grant, 1981). These directedforgetting results have been interpreted to suggest that subjects must actively maintain a to-be-remembered stimulus, as failure to do so in the "forget" condition results in disrupted performance on probe retention tests.

The primacy portion of the serial position curve has frequently been interpreted as reflecting the effects of rehearsal in working memory (contributing to storage in longterm memory). Consequently, findings of primacy and recency in serial position curves from pigeons and monkeys have been taken as evidence that these species rehearse in their visual working memory (see discussion and rebuttal by Sands, Urcuioli, Wright, \& Santiago, 1984).

Fourth, Wagner (1978) noted that animals learn and remember novel or surprising stimuli better than expected and familiar stimuli. This finding was interpreted to suggest that novel and surprising stimuli elicit attention and require active rehearsal.
However, each of these demonstrations has also been subject to criticism. In the distractor paradigm, for example, it is difficult to determine whether the intervening task literally distracts the subject, preventing active maintenance rehearsal, or whether it provides stimulation that displaces or interferes with the memory retained passively in the visuospatial sketchpad. Similarly, interpretation of directed forgetting results has been disputed (see Macphail, 1986, and Wixted, 1989, discussed below). Baddeley (1986) discussed problems associated with interpreting either the primacy or the recency portions of the serial position curve as evidence for rehearsal and concluded that the effects of surprising stimuli might be accounted for by attentional factors at the encoding, versus rehearsing, stage.

The present experiments were designed to compare the alternative interpretations of some of these phenomena. It was hoped that the studies would reveal the nature of the maintenance or rote rehearsal, defined for this study as a process that is actively used to refresh the short-term memory of information that is difficult to recode verbally and for which there is no representation in long-term memory. Using several different paradigms, we anticipated that variables that influence or fail to influence rehearsal would be distinguished, and that these findings would suggest whether such rehearsal is characteristically visual or spatial, voluntary or obligatory, rapid or slow, and effective or ineffective. Nonhuman primates as well as humans were tested to permit identification of interactions between verbal and visuospatial working memory systems.

\section{EXPERIMENT 1 Distractor Paradigm}

A common method for studying verbal working memory is to identify the types of distractor activity that disrupt recognition or recall. Tracking tasks have been shown to effectively disrupt visuospatial but not verbal working memory (see, e.g., Baddeley, 1986; Morris, 1989). Consequently, a distractor paradigm with a tracking task was employed in this experiment. It was hypothesized that pursuit tracking during a retention interval would prevent subjects from rehearsing, resulting in compromised recognition performance.

\section{Method}

Subjects. Four rhesus monkeys (Macaca mulatta; age range 2-11 years; 3 males) were tested in this experiment. The training and test histories for 2 of these animals (Abel and Baker) have been extensively described elsewhere (e.g., Rumbaugh, Richardson, Washburn, Savage-Rumbaugh, \& Hopkins, 1989). The other 2 monkeys were trained with similar methods to manipulate a joystick in order to respond to computer-generated stimuli (see Washburn \& Rumbaugh, 1994). All monkeys had extensive experience with memory tasks, including delayed matching to sample, prior to this study. Each monkey was singly housed and was not restrained or deprived of food or water during testing. Twenty human subjects were also tested in this experiment. These undergraduate students (age range 18-34 years; 14 females) volunteered to participate in exchange for class credit.

Apparatus. All subjects were tested with the Language Research Center's Computerized Test System (LRC-CTS; Rumbaugh et al., 

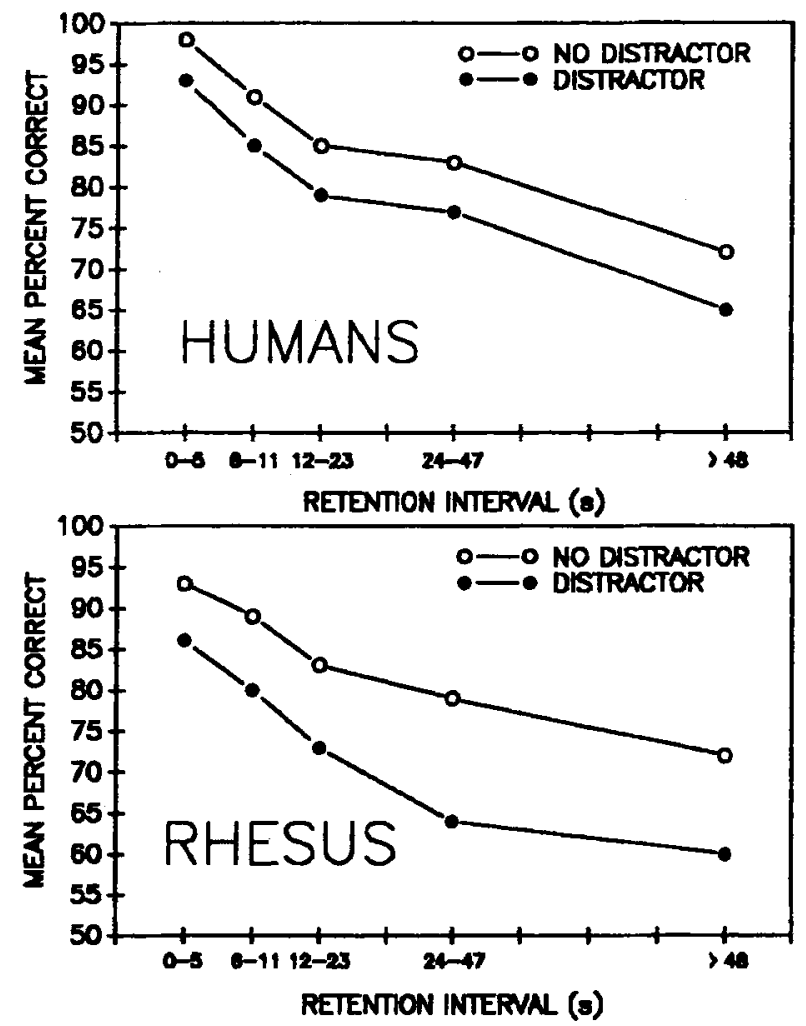

Figure 1. Experiment 1: Mean percentage of correct recognition as a function of retention interval and distractor condition for humans and rhesus monkeys.

1989). A 386-based computer was used to generate and present stimuli on a color monitor. Subjects responded to these stimuli by manipulating a joystick, which in turn determined the movements of a cursor (a $1.25 \times 1.25 \mathrm{~cm}$ plus sign) on the screen at a speed of $5 \mathrm{~cm} / \mathrm{sec}$. Auditory feedback was provided through the computer's internal speaker or via an external speaker/amplifier. For the monkeys, correct responses resulted in the delivery of a $97-\mathrm{mg}$, fruit-flavored chow pellet (P. J. Noyes) using a Gerbrands 5150 dispenser and a Metrabyte interface (ERA-01).

Task. A delayed matching-to-sample (DMTS) task was written for this experiment. Each trial began with the cursor presented in the middle of the screen. A $4 \times 3$ cell checkerboard stimulus, filled at random with colors and patterns, was presented in random position on the screen (but not within $5 \mathrm{~cm}$ of the cursor). The checkerboard stimuli measured $2.5 \times 5 \mathrm{~cm}$, with each of the 12 cells filled with a randomly selected color and pattern.

Each trial was subject initiated to provide ample opportunity to encode target stimuli. To initiate a trial, a subject was required to manipulate the joystick so as to bring the cursor into contact with this sample stimulus, whereupon the sample would disappear for $0,6,12$, 24 , or $48 \mathrm{sec}$ (nominal; see below). Following this retention interval, two comparison stimuli were presented on the screen, again in randomly selected but nonoverlapping positions and separated from the cursor by at least $5 \mathrm{~cm}$. One of these comparison stimuli was identical to the sample, whereas the other differed in terms of the contents of one or more cells. If the subject brought the cursor into contact with the stimulus that matched the sample, a rising tone sequence was presented and, for the monkeys, a pellet reward was delivered. Touching the nonmatching comparison stimulus with the cursor resulted in a raucous buzz. In either case, a 5-sec interval separated each trial.
The DMTS task was administered under two conditions. In one condition (baseline), the cursor remained on the screen during each retention interval in the position it occupied when it was in contact with the sample stimulus. In this condition, subjects could only wait for the presentation of the comparison stimuli to respond. In the second condition (distractor), a white circle, $5 \mathrm{~cm}$ in diameter, replaced the sample stimulus immediately when it was touched by the cursor. This circle moved diagonally across the screen at a speed of $2.5 \mathrm{~cm} / \mathrm{sec}$, "bouncing" off each border. Subjects were required to maintain unbroken contact with this moving circle for the duration of the retention interval. To ensure that subjects were attending to this pursuittracking distractor task, errors (allowing the cursor to go beyond the circumference of the circle) reset the timer and thus lengthened the retention interval. Only after the subject continuously tracked the circle for the appropriate time were the comparison stimuli presented for the recognition portion of the trial.

Procedure. Each subject was tested on alternating blocks of distractor and baseline trials, with the actual retention intervals for each baseline trial yoked to a corresponding retention interval ( $0-48 \mathrm{sec}$ plus any penalty for tracking errors) in the distractor condition. By yoking trials, it was ensured that the duration of retention intervals was exactly matched between trials in the baseline and the distractor conditions.

Each human subject completed five blocks of 10 trials in each condition (100 trials total per subject). Each monkey completed 25 blocks of 10 trials for each condition ( 500 trials per subject). Each monkey performed 100 DMTS trials per day. For the remainder of each test day, the monkeys were tested on other, unrelated tasks (see Washburn \& Rumbaugh, 1994).

\section{Results}

Because retention intervals could vary from their nominal values, trials were partitioned into five class intervals according to retention interval: $0-5 \mathrm{sec}, 6-11 \mathrm{sec}, 12-23 \mathrm{sec}$, 24-47 sec, and $48 \mathrm{sec}$ or longer. Separate analyses were conducted for the two species. For human subjects, accuracy of responding was analyzed as a function of condition (baseline vs. distractor) and retention interval. Recognition was significantly affected by both independent variables $[F(1,19)=7.33, p<.05$, and $F(4,76)=21.59$, $p<.01$, respectively]. No interaction of the terms was observed. As can be seen in the top panel of Figure 1, recognition was reliably worse in the distractor condition across retention intervals.

Comparable findings were obtained for rhesus monkeys (see Figure 1, bottom panel). Performance was significantly poorer with longer retention intervals $[F(4,12)=$ $12.17, p<.01]$ and was reliably compromised in the distractor condition $[F(1,4)=15.96, p<.05]$. No interaction of the variables was found.

\section{Discussion}

At first glance, these data seem to suggest that humans and rhesus monkeys do rehearse visuospatial information in working memory unless prevented from doing so by a distractor activity. Certainly the present data are comparable to other published effects of distractor task-for verbal memory in humans as well as in studies of visual memory in nonhuman animals - that have been interpreted as evidence for nonverbal rehearsal. Given these results, we were prepared to manipulate other variables (e.g., tracking 
difficulty, the amount of spatial vs. visual distraction) to elucidate the nature of such rehearsal.

However, an alternate interpretation is suggested by the data. Perhaps the appearance of the tracking circle itself interfered with the memory of a target stimulus, rather than the activity of tracking, preventing the subjects from maintenance rehearsal. On the basis of findings that irrelevant and "unattended" pictures presented during retention intervals reliably disrupt visual working memory, Baddeley (1986) suggested that visual stimuli have obligatory access to the visuospatial sketchpad. Support for this interpretation is gleaned from the absence of a retention interval $\times$ condition interaction for either species in the present data. We anticipated that the forgetting curve would be steeper in the absence of rehearsal relative to that in the baseline condition.

In particular, note that performance was worse in the distractor condition than the baseline condition even after a 0 -sec retention interval. On baseline trials in the 0 -sec condition, contact between the cursor and the sample stimulus caused the sample to be replaced by the tracking circle, which was then immediately removed for the recognition portion of the experiment. There was no time for movement of the target or cursor (or for tracking errors); that is, subjects did not actually track at all in the 0 -sec retention interval condition. Nevertheless, recognition was disrupted in the distractor condition - and the degree of disruption was approximately the same as during other retention intervals.

Disrupted performance at the 0 -sec retention intervals suggests that the tracking task may not have distracted the subjects from rehearsing at all. Rather, the tracking circle may have caused interference (i.e., compromised the representation of the sample in working memory) that resulted in relatively poor retention across delay intervals. To test this interpretation, a second experiment was conducted.

\section{EXPERIMENT 2 Distractor-Task Paradigm}

\section{Method}

Twenty human volunteers were tested. None of these subjects (age range 18-37 years; 14 females) had participated in Experiment 1. However, the 4 rhesus monkeys from that study were again included. Each subject was tested with the task and apparatus from the previous study.

Each human subject completed eight blocks of 10 trials in each condition (160 trials total per subject). Each monkey completed 30 blocks of 10 trials for each condition (600 trials per subject). Once again, retention intervals were yoked between the conditions (baseline vs. tracking) and were grouped into class intervals for analysis. For the present experiment, however, a new condition was added to the task. On half of the distractor-condition blocks of trials, the tracking circle was flashed on the screen for $500 \mathrm{msec}$ once the sample was removed. No tracking was required on these trials (hereafter termed the "interference condition"), but tracking of the moving circle was required on the other half of the distractor-condition trials, as in Experiment 1.

The purpose of this manipulation was to dissociate the amount of compromise in performance due to interference from that due to dis- traction from rehearsal per se. It was hypothesized that performance would be compromised when rehearsal was prevented by tracking. For this effect to be interpreted as evidence for rehearsal, however, performance in the distractor condition should also be significantly worse than in the interference condition (which should not prevent the subjects from rehearsing).

\section{Results}

The accuracy of responding was analyzed separately for each species using retention interval and condition (baseline, distractor, and interference) as independent variables. For human subjects, significant main effects were observed for retention interval $[F(4,76)=42.74, p<.01]$ and distractor condition $[F(2,38)=36.55, p<.01]$. Post hoc analysis revealed that the accuracy of recognition was significantly highest in the baseline condition $(M=88 \%)$, but did not differ between the distractor and interference conditions $(79 \%$ and $80 \%$, respectively; HSD $=7 \%)$. This effect is evident in Figure 2.

For rhesus monkeys, significant effects were found for retention interval $[F(4,12)=45.19, p<.01]$ and distractor condition $[F(2,6)=22.88, p<.01]$. Again, performance in the distractor condition $(M=76 \%)$ did not differ from performance in the interference condition $(78 \%)$, whereas both differed from baseline accuracy $(85 \%$; HSD $=7 \%)$. This effect is also depicted in Figure 2.
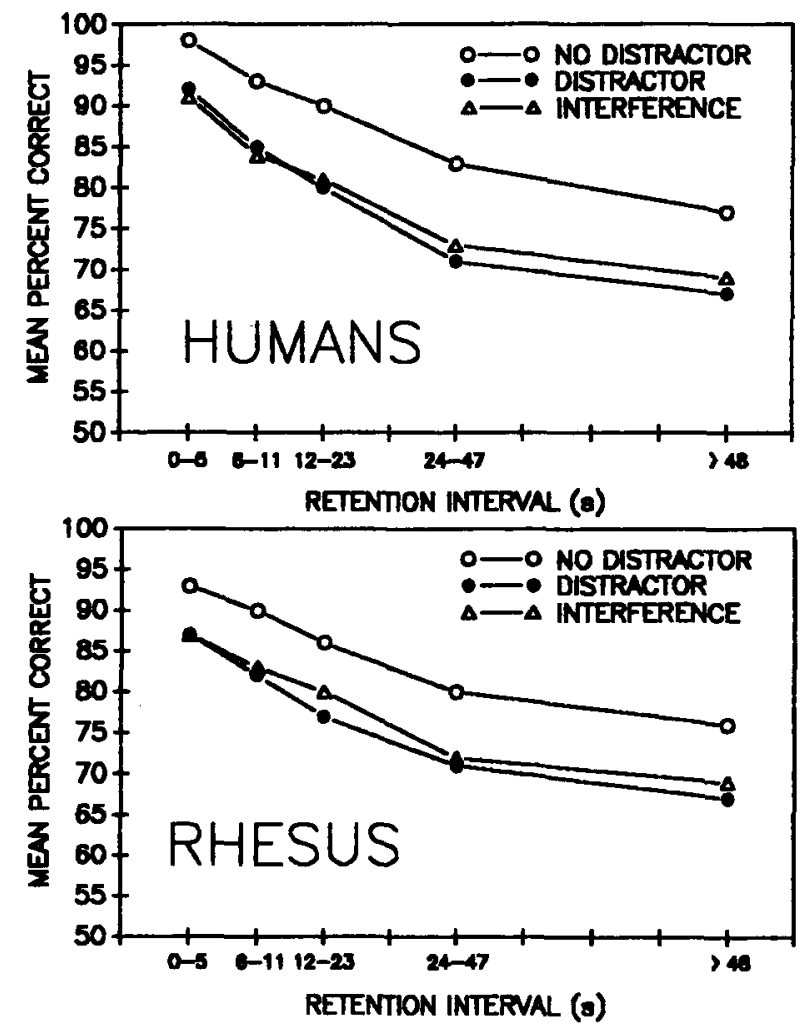

Figure 2. Experiment 2: Mean percentage of correct recognition as a function of retention interval and distractor condition for humans and rhesus monkeys. 


\section{Discussion}

Comparable forgetting was produced when subjects were required to track the movements of a circle on the screen, which was presumed to prevent the subjects from maintenance rehearsal, as when they merely saw the circle flashed on the screen. Passive observation of this circle should not have prohibited rehearsal, but would be expected to produce displacement or interference. Notably, the prevention of rehearsal did not result in more forgetting than was observed for interference.

That these findings reflect memory rather than encoding or other perceptual process is suggested by the sequence of each trial. In all conditions, the sample stimulus remained visible until the subject chose to make it disappear. Only after this event, which operationalizes the point at which the sample was seen and encoded, did the tracking circle appear (or flash). One might argue that encoding could continue after the stimulus was removed, but it is unclear why this would happen with indefinitely available stimuli except as a means of rehearsal.

Rather, these results and those from Experiment 1 suggest that the presentation of an irrelevant visual form (the tracking circle) after removal of the sample stimulus interfered with memory for the sample (see Toms, Morris, \& Foley, 1994, for a similar finding). Thus, the distractor condition appears not to have involved actual distraction at all. Rather, the tracking circle appears to have produced an effect on memory, both for humans and monkeys, much like the suffix effect that has been reported for verbal memory (see Baddeley, 1986). This finding that visual memory is subject to interference is also consistent with reports that visuospatial memory is poor for more than one item (Phillips \& Christie, 1977a, 1977b). However, it is not consistent with the suggestion that humans and monkeys actively rehearse nonverbal information during retention intervals.

\section{EXPERIMENT 3 Directed Forgetting Paradigm}

There are two other interpretations of these findings. First, it may be that tracking does not preclude nonverbal rehearsal. Just as verbal rehearsal is prevented by tasks that require vocal or subvocal articulation, the tracking activity was selected because it is an attention-demanding visuospatial task. Nonetheless, it is impossible to discriminate whether the task fails to disrupt rehearsal or whether there is no rehearsal to be prevented.

Conversely, it might be suggested that visual memory is compromised both by interference/displacement and distraction (i.e., prevention of active maintenance rehearsal), but that the effects of these factors are not additive. That is, subjects may actively rehearse nonverbal information unless rehearsal is prevented or the memory is impugned by the presentation of irrelevant stimuli. Taken together, these two alternative interpretations suggest that one needs to look for evidence regarding the nature of nonverbal rehearsal using a paradigm in which effects are not masked by interference.
One such procedure is the instructed forgetting paradigm, in which removal of the sample stimulus (or stimuli) is followed by a cue that alerts the subject whether or not a retention test will follow. On half of the trials, the subject is prompted that there will be a recognition or recall test-and thus that the subject should try to remember the sample. On the remaining trials, the cue indicates that no test will follow and that the trial will end after the retention interval. On a small proportion of these forget trials, however, a retention test is given. Performance on these probe trials, during which the subject was presumably not trying to rehearse, is compared with performance on the remember trials. Note that the to-be-remembered stimulus is followed by a cue in both conditions; thus, differences between the conditions cannot be explained by interference or displacement, as was true for Experiments 1 and 2.

Although human subjects have been tested under conditions of instructed forgetting (Basden, Basden, \& Gargano, 1993; Bjork, 1989), the paradigm is in many ways ideally suited for research with nonhuman animals. First, nonhumans are unlikely to suspect the purpose of the study and bias their responses with these demand characteristics. Second, nonhuman animals can perform the thousands of trials necessary to produce a sufficient number of lowprobability probe trials for analysis (see below). For these reasons, Experiment 3 was conducted only with rhesus monkey subjects.

One problem with the instructed forgetting task was described by Kendrick et al. (1981). The advantage for retention in the remember condition as compared with probe performance from the forget trials appears to disappear if the animals have the opportunity for reward following the forget trials. That is, pigeons recognize sample stimuli just as accurately following the forget cue as following the remember cue if they have the opportunity to make an irrelevant response that will be reinforced. Kendrick et al. concluded that whereas three of their experiments supported a rehearsal interpretation, the fourth study suggested a context effect on retention - a finding consistent with Wixted's (1989) review. Wixted interpreted all instructed forgetting effects as evidence for stimulus control by the serial compound (sample + cue) of responding rather than for active poststimulus rehearsal.

For several reasons it seemed reasonable, however, to attempt a directed forgetting experiment with monkeys. Kendrick et al. (1981) studied DMTS performance by pigeons with short $(4 \mathrm{sec})$ retention intervals. More importantly, those authors reported differential rates of keypecking during retention intervals for the directed forgetting and directed remembering conditions. These differences in poststimulus behavior confounded the cue and context on which the pigeons responded. That is, the behavior itself may provide a cue for responding (e.g., peck the nearest key) quite independently of maintenance rehearsal. In contrast, the DMTS task used with the monkeys (in which stimulus positions were randomized) permitted no opportunity for overt, rehearsal-like behavior. Rather, only the 
Table 1

Conditions of Experiment 3

\begin{tabular}{lccc} 
& \multicolumn{3}{c}{ Cue } \\
\cline { 2 - 4 } Test & $\begin{array}{c}\text { Remember } \\
\text { (DMTS Expected) }\end{array}$ & $\begin{array}{c}\text { Forget } \\
\text { (MTS Expected) }\end{array}$ & $\begin{array}{c}\text { Forget } \\
\text { (No Task Expected) }\end{array}$ \\
\hline DMTS & D-D & M-D* & N-D* \\
MTS & D-M* & M-M & N-M* \\
\hline
\end{tabular}

Note-Conditions have been named by abbreviating the cue condition (i.e., the test that was expected) and the test that was actually administered (D, DMTS; M, MTS; N, no task). DMTS, delayed matching-tosample; MTS, matching-to-sample. *Probe trials occurring with $p=$ .10 within a block of trials.

existence or absence of active, covert rehearsal should differentiate the forget-and remember-cued trials.

\section{Method}

Subjects. The 4 monkeys from the previous study were used in Experiment 3 as well as 2 additional rhesus monkeys (both 9-yearold males). The latter animals were joystick-trained using the same procedures as the other monkeys, but their pursuit-tracking skills were not sufficient to compel inclusion in the first two experiments. All monkeys had extensive experience with memory tasks, including DMTS, prior to this study. All monkeys were tested in their home cages and were not deprived of food or water for purposes of testing.

Apparatus and tasks. The monkeys were tested with the LRCCTS, described earlier. Each monkey performed the DMTS task (used in the baseline condition of Experiment 1) with 0- to 48-sec retention intervals. Type of cue (remember vs. forget), type of test (expected vs. surprise), and task type (DMTS, matching-to-sample [MTS], or no task) were manipulated across three blocks of 2,000 trials. As summarized in Table 1, this design yields six critical conditions. In one block, subjects received either remember-cued trials (indicating that a DMTS test would follow the retention interval) or forget-cued trials (indicating that the trial would end immediately after the retention interval). On $10 \%$ of the forget-cued trials, a surprise DMTS recognition test was given (N-D condition). Note that these conditions are designated by the test that was expected $(\mathrm{D}=$ DMTS, $M=$ MTS, $N=$ no test) and the test that was administered (e.g., N-D is a DMTS test when no test was cued). On $10 \%$ of the remember-cued trials, a surprise MTS test was given (i.e., the sample appeared simultaneously with the comparison stimuli; D-M condition). The latter condition permits the level of working memory decrements (from D-D trials) to be distinguished from psychomotor or other errors.

In the second block of trials, subjects again received remembercued trials (D-D condition and D-M condition) or received a different forget cue that signaled an MTS test after the retention interval (M-M condition). On $10 \%$ of these trials, however, the sample stimulus was not presented simultaneously with the comparison stimuli, resulting in a surprise probe test (M-D condition).

In the third block of trials, subjects received either remember-cued trials (D-D condition and D-M condition) or forget-cued trials from the first block of trials (i.e., trials ended without reinforcement after the retention interval). On $10 \%$ of these forget-cued trials, a surprise MTS test was administered ( $\mathrm{N}-\mathrm{M}$ condition).

For each condition, the color of the screen during the retention interval (magenta, blue, or white) signaled the type of trial. Note that this cue was presented only after the sample was removed; thus, any differences in retention could not have stemmed from motivation or other differences in the perception or encoding of the sample stimulus. To prevent color-specific interference biases, the specific screen color that signaled each of the three trial types was randomized for each animal. Trial type was randomized across trials within each block.
Across 2,000 trial blocks, each monkey produced an average of 100 trials in each of the probe conditions (N-D, M-D, N-M, and D-M conditions). This low rate of probe trials attenuated the likelihood that the monkeys would treat the forget-cued trial types like the remember-cued trials. This rather complicated experimental design was used to afford three critical comparisons. Significantly better performance in the D-D condition than the N-D performance would replicate other directed forgetting evidence for rehearsal by animals. On the other hand, significantly better performance in the M-D than in the N-D condition would suggest that the directed forgetting effect is an artifact of reinforcement conditions, as suggested by Kendrick et al. (1981). That is, this effect would indicate that performance is compromised whenever reinforcement is not anticipated, irrespective of whether the subject is cued to rehearse. Finally, a significant difference between performance in the N-M condition and in the D-M condition would suggest that the directed forgetting phenomenon is an artifact reflecting the secondary punitive properties of the "no task" cue.

\section{Results}

Recognition accuracy was significantly influenced by retention interval $[F(4,20)=14.41, p<.01]$. On the average, recognition accuracy was $94 \%$ after 0 -sec retention intervals, $87 \%$ after 6 -sec, $80 \%$ after 12 -sec, $75 \%$ after 24 -sec, and $65 \%$ after $48-\mathrm{sec}$ retention intervals.

Recognition accuracy was also analyzed as a function of task type (DMTS vs. MTS) and expected test (DMTS, MTS, and none). Significant main effects were found for both variables $[F(1,5)=62.05, p<.01$, and $F(2,10)=$ $228.91, p<.01$, respectively]. Responses were significantly more accurate on MTS than on DMTS trials and were significantly compromised when subjects were expecting no test at all. These variables also interacted significantly $[F(2,10)=38.25, p<.01]$. As noted, three planned post hoc comparisons were made using the Tukey HSD test $(\mathrm{HSD}=5 \%)$ and the means reflected in Table 2 .

\section{Discussion}

Subjects performed significantly better on D-D trials, cued with a remember signal, than on forget-cued N-D trials. Typically, this effect is interpreted as evidence for purposive, active maintenance rehearsal following a remember cue. However, M-D trials, which were signaled by a cue that should mean "there is no need to rehearse on this trial because the sample will reappear with the comparison stimuli" also produced significantly better performance than did N-D trials. Thus, the monkeys performed relatively (and comparably) well on all trials for which they expected the opportunity for reinforcement. Still, what is the mechanism for disrupted performance on forget-cued trials? Performance on the N-M trials, which was significantly worse than for other MTS test conditions, suggests

Table 2

Results of Experiment 3 (in Percent)

\begin{tabular}{lccc} 
& \multicolumn{3}{c}{ Cue } \\
\cline { 2 - 4 } Test & $\begin{array}{c}\text { Remember } \\
\text { (DMTS Expected) }\end{array}$ & $\begin{array}{c}\text { Forget } \\
\text { (MTS Expected) }\end{array}$ & $\begin{array}{c}\text { Forget } \\
\text { (No Task Expected) }\end{array}$ \\
\hline DMTS & 79 & 80 & 55 \\
MTS & 94 & 95 & 85 \\
\hline
\end{tabular}


the answer. The forget cue, typically associated with the end of an unreinforced trial, appears to acquire secondary punitive properties. Subjects touch the sample and see the forget cue, which decreases the likelihood of touching that stimulus again on DMTS or even MTS probe trials! In other words, the monkeys treat N-D and N-M trials as if they were discrimination learning opportunities rather than recognition tests and avoid the stimulus that recently resulted in the aversive forget cue.

Two possible interpretations exist for these findings. First, it is possible that the monkeys cannot or do not rehearse under any conditions. Rather, they avoid the sample in the forget conditions, as suggested by Wixted, 1989, in his stimulus-control account. This interpretation is also consistent with the conclusion by Bjork (1989) that forgetcued stimuli are inhibited. In either case, directed forgetting effects do not support a rehearsal explanation. Further, it is noteworthy that performance in the remember conditions was in fact comparable to levels of accuracy in the baseline condition of Experiments 1 and 2. This suggests the poststimulus color cues did not themselves interfere with visual working memory.

However, an alternative interpretation is possible. It is possible that the monkeys do rehearse, but that rehearsal is obligatory. Following this suggestion, performance may have been comparable in the D-D and M-D conditions because the monkeys rehearse even when they do not need to do so. Of course, one would expect obligatory rehearsal to benefit forget-cued trials as well; however, the tendency to avoid the sample on these N-D and N-M trials may have counteracted any maintenance rehearsal.

\section{EXPERIMENT 4 Dual-Task Paradigm}

Experiments 1, 2, and 3 yield one of two accounts of active maintenance rehearsal of nonverbal stimuli by humans and monkeys: Either rehearsal is an obligatory process that is disrupted by intervening tasks in a way that is not additive with the effects of interference by irrelevant stimuli, or rehearsal is not used to support short-term recognition of visual forms at all. These experiments emphasize the difficulty of studying nonverbal rehearsal using the techniques described in the literature. One must infer the existence of active, covert processing designed to maintain information in working memory, but attempts to measure this processing either interfere with the rehearsal or may miss it altogether. Perhaps a new paradigm is needed to address the issue.

Actually, the procedure utilized in Experiment 4 is hardly novel, but it has not before been applied to the particular question of determining whether humans and monkeys rehearse during retention intervals. A dual-task procedure, common in studies of attention, was developed for this experiment. Innumerable researchers have reported that the time to perform a secondary task (and the error rate during its execution) is increased when the task is performed concurrently with another, primary task as compared with when the secondary task is performed alone. For example, the time required to detect and respond to a tone stimulus is longer if subjects are performing a concurrent tracking task than if the subjects are performing only the tone-detection task. In this case, the primary task (tracking) consumes resources, resulting in less attention for (or necessitating a shift of attention to) the secondary (tone) task. In the absence of the primary task, the full capacity of attentional resources is available for the tone-detection task.

In terms of the present question, one might hypothesize that response times on a probe detection task would be reliably longer if subjects performed the task while rehearsing than if their attention was not occupied with active, covert rehearsal. Alternatively, failure to find relatively long response times to probe stimuli presented during retention intervals would suggest that the subjects are not otherwise involved in an attention-demanding activity (namely rehearsal). Of course, this probe task might itself disrupt rehearsal, causing interference, as in Experiment 1 . In the present experiment, however, such interference is inconsequential because the existence or absence of active rehearsal would be reflected in the response times to the probe task.

\section{Method}

Subjects. Fifty volunteers from the undergraduate subject pool at Georgia State University participated in this study. These students (age range 18-43 years; 33 females) received class credit for their service.

The same 6 rhesus monkeys that served in Experiment 3 were also tested in the present study. Although each of the monkeys had prior joystick training, none had been trained to execute a response (e.g., press a button) in a tone-detection task. However, each monkey had performed a relative numerousness task prior to this study and thus could already perform the numeric probe task employed here (see below; Washburn \& Rumbaugh, 1991). The monkeys received pellet reinforcers for each correct response, but received supplemental food irrespective of their task performance.

Apparatus and task. The apparatus for Experiment 4 was the same as in the previous experiments, but the DMTS task was altered in several ways. Essentially, the task resembled the baseline condition from Experiment 1 . However, a secondary task demand was added. During the retention interval of $10 \%$ of the trials, the screen was suddenly cleared and the cursor was presented in the middle of the screen, flanked by a 2 and a 1 . The numerals were white characters, $2.5 \times 2.5 \mathrm{~cm}$, and were positioned randomly $2.5 \mathrm{~cm}$ to the left and right of the cursor. This screen configuration was maintained until the subject manipulated the joystick left or right, which caused the cursor to immediately contact the corresponding numeral. When a response had been made to the probe stimuli, the screen was cleared and the cursor was returned to the position it occupied when it had contacted the target stimulus, where it stayed for the balance of the retention interval. Subsequently, comparison stimuli were presented as in the baseline condition.

On $10 \%$ of the trials, this probe stimulus was suddenly presented during the intertrial interval-after responses to the comparison stimuli had been obtained and while the subject awaited the next sample stimulus. Again, the intertrial interval continued only after the subject had selected either the 2 or the 1 in the probe display. 
Table 3

Results of Experiment 4: Recognition Accuracy and Probe Response Times (RTs, in Milliseconds)

\begin{tabular}{|c|c|c|c|c|c|c|}
\hline \multirow[b]{3}{*}{ Group } & \multirow{3}{*}{$\begin{array}{l}\text { Stimulus } \\
\text { Type }\end{array}$} & \multirow{3}{*}{$\begin{array}{c}\text { No } \\
\text { Probe }(\%)\end{array}$} & \multicolumn{4}{|c|}{ Interval } \\
\hline & & & \multicolumn{2}{|c|}{ Retention } & \multicolumn{2}{|c|}{ Intertrial } \\
\hline & & & $\%$ & RT & $\%$ & RT \\
\hline Humans & words & 88 & 84 & 909 & 88 & 844 \\
\hline Humans & forms & 90 & 79 & 840 & 86 & 863 \\
\hline Monkeys & forms & 86 & 73 & 586 & 85 & 575 \\
\hline
\end{tabular}

Irrespective of when the probe was presented, response time was measured from the appearance of the probe screen until a response (collision between the cursor and the 1 or 2 ).

An additional change to the DMTS task involved the stimuli used. On half of the trials, the checkerboard stimuli described in the earlier experiments were used. On the remaining trials, subjects were presented with a string of seven randomly selected, nonrepeating digits or consonants to remember (see Procedure). For example, a string of seven digits (e.g., 8572301) was presented in random position on the screen, to which the subject moved the cursor. The digits disappeared and the cursor remained visible during the retention interval (during which time the probe stimuli may have appeared). Subsequently, two strings of digits were presented in random positions. One of the strings matched the sample exactly, whereas the digits in two positions were transposed in the other comparison stimulus (e.g., 8573201). After the subject brought the cursor into contact with one of the stimuli, feedback was provided according to the accuracy of recognition and an intertrial interval ensued (during which time the probe stimuli may have appeared).

Procedure. Two groups of human subjects were tested. Twentyfive subjects were tested with checkerboard forms and digit strings as stimuli, with stimulus type randomly determined each trial. The second group of subjects received forms and consonant strings as stimuli, randomly determined for each trial. The purpose of this manipulation was to evaluate the possibility of numeral-specific interference between the sample string and the probe stimuli that may have biased the probe response times. All human subjects were given instructions describing the DMTS procedure and indicating that occasionally a 2 and a 1 would appear together on the screen. Subjects were told that any time this probe screen was presented, they should immediately stop whatever they were doing, touch the 2 with the cursor as quickly as possible, and then return to their previous activity. The instructions for Group 1 clearly distinguished between the 2 and 1 of the probe screen and any 2 or 1 in the sample stimulus.

Each human subject completed 11 blocks of 10 trials (the first block for practice) with an opportunity for rest between each block. Thus, an average of 10 trials ( 5 for verbal stimuli, 5 for form stimuli) was obtained from each subject for probe trials presented randomly during the retention interval, and 10 trials were obtained for probes presented randomly during the intertrial interval. This low prevalence of probes was used to encourage subjects to concentrate on the DMTS task throughout, rather than dividing attention between DMTS and probe detection. Every trial had a $10-\mathrm{sec}$ retention interval and a 10-sec intertrial interval. When it occurred at all, the probe screen was presented at a random point within either the 10sec retention interval or the 10 -sec intertrial interval.

After completing the experiment, each subject was given a debriefing questionnaire that provided more details about the experiment. On the survey, the subjects reported the frequency, ease, and strategy with which they rehearsed (or did not rehearse) the verbal stimuli (digits or consonants) and the visual forms.

The rhesus monkeys were also tested on the DMTS task with occasional secondary task probes. Obviously, the monkeys could not be instructed to respond to these probes quickly. However, prior studies indicate that the monkeys do respond as quickly as possible (and usually much quicker than humans!) without additional instruction. For example, their chronometric data have produced evidence of Stroop-like interference and facilitation, symbolic distance effects, functional cerebral asymmetries, and popout effects in visual search (see, e.g., Hopkins, Washburn, \& Rumbaugh, 1990; Washburn, 1994). All trials for the monkeys utilized checkerboard stimuli rather than consonant or digit strings. Each monkey performed 1,000 trials, providing an average of 100 response times per animal for probes presented during the retention interval and 100 response times per animal for probes presented during the intertrial interval.

\section{Results}

Separate analyses of variance were performed for each species and for each dependent measure (accuracy on the DMTS task and response time on the probe task) with two within-subjects variables. For the humans, one betweengroups variable (digits vs. consonants) was also included as a factor, but because the group variable was inconsequential in all analyses, all results will be discussed across groups. For the humans, no main effects were observed in the accuracy data for stimulus type (visuospatial vs. verbal) or for probe interval (retention interval vs. intertrial interval), nor did the two variables interact. Accuracy was generally high for all conditions (Table 3).

Analysis of probe response times produced a different pattern of results. A stimulus type $\times$ probe interval interaction was observed $[F(1,49)=6.29, p<.05]$. Response times to probes that were presented during the retention interval of trials in which the sample stimulus was verbal (consonants or digits) were significantly longer than the probe response times in any other condition (HSD = $63 \mathrm{msec}$; see Table 3 ). No other mean response times were found to differ from one another.

No statistically significant effects were obtained in the analysis of the monkeys' DMTS accuracy or probe response time. As can be seen in Table 3, mean accuracy was comparable across conditions, as were the response times to probe stimuli presented during the retention or intertrial intervals.

Descriptive analysis of the debriefing questionnaires revealed that most humans $(96 \%)$ frequently rehearsed the verbal stimuli and that they found the rehearsal easy to do. In contrast, only $20 \%$ of the subjects claimed to have rehearsed the nonverbal stimuli. In their descriptions of rehearsal, it was clear that these subjects attempted to recode verbally each of the visuospatial sample stimuli and rehearsed verbally. No subject reported rehearsing either type of stimulus during the intertrial intervals. These reports provide mere anecdotal evidence, although it is interesting that they are well corroborated by the chronometric findings.

\section{Discussion}

It is clear what these data should look like if rehearsal exists, because response times were significantly longer to probe stimuli that were presented while human subjects rehearsed the verbal stimuli than when presented during the intertrial interval while the subjects were not actively re- 
hearsing. Conversely, the absence of any difference in the probe response times for visuospatial stimuli suggests that the humans were not engaged in activity during the retention interval of trials in which nonverbal stimuli were to be remembered. A similar finding was apparent for the rhesus monkeys, whose response times to the secondary task were comparable irrespective of whether the probes interrupted a retention interval or an intertrial interval. The fact that response times to the probe screen were similar to those reported previously for humans and monkeys on a simple relative numerousness task further suggests that no ongoing activity was consuming attentional resources that might otherwise have competed with secondary task performance. In sum, the data provide no evidence for rehearsal by monkeys or for nonverbal rehearsal by human subjects. In contrast, response times in the hypothesized direction support the existence and use of active verbal rehearsal by humans for maintaining digits or consonants in working memory.

It might be argued that the secondary task used in this experiment (i.e., responding to the 2 in a $2-1$ or 1-2 probe) did not require common attentional resources with maintenance rehearsal of visual forms (although the task also had a spatial component in that subjects had to determine whether the 2 was on the left or the right), and that this is why no response time differences were observed. It is true that no clear overlap existed between rehearsal of checkerboard images and the judgment task. On the other hand, no a priori overlap existed between consonant strings and the judgment task, but dual-task complementarity was observed in the response time data for these stimuli. Additionally, the probe task did compromise primary task performance (i.e., recognition in the DMTS task). In any case, it would be circular and unparsimonious to postulate separate pools of attentional resources to account for these effects (or their absence).

\section{GENERAL DISCUSSION}

This series of experiments was designed to elucidate the nature of maintenance rehearsal in nonverbal working memory. The paradigms were selected because they had been used in prior research to study nonverbal rehearsal. We have attempted to find variables that systematically affect retention, looking for dissociations or convergent indices of the characteristics of nonverbal rehearsal. The findings suggest that maintenance rehearsal of visuospatial stimuli (1) is interrupted by intervening tasks, (2) but not in a way that is additive with interference effects; (3) is obligatory, even under conditions in which rehearsal is not necessary; (4) requires no attentional resources, or at least different resources than those that are required for numer$\mathrm{ical} /$ spatial judgments or verbal rehearsal; and (5) appears to be comparable for humans and rhesus monkeys.

Alternatively, one might dismiss the present findings altogether, suggesting that the tasks, variables, manipulations, or subjects are irrelevant to nonverbal rehearsal. Such an argument would fail to recognize that each of these elements was selected, on the basis of published findings, to maximize the likelihood that rehearsal-specific alterations in nonverbal working memory could be identified.

A third, simpler interpretation seems more plausible. In these four experiments, no evidence was found for the existence of active maintenance rehearsal in visuospatial working memory. Additionally, the previous reports of nonverbal rehearsal (see Rilling \& Neiworth, 1986; Roitblat, 1987), which themselves were already equivocal for reasons discussed earlier, are seriously challenged by the present findings in that alternative interpretations (i.e., interference, conditioning) of each phenomenon (i.e., distractor-task effects, directed forgetting) have been specifically supported. Humans and nonhuman primates alike appear to have retained visual information in working memory quite effectively - unless other stimuli were permitted to interfere with or displace the to-be-remembered material - without maintenance rehearsal. In contrast to verbal rehearsal by humans, which can be reliably demonstrated either directly or indirectly, as in Experiment 4, support for the existence of an active nonverbal maintenance rehearsal process remains elusive.

Clearly, there are several reasons why this might be so. The "lack of evidence for nonverbal rehearsal" is not the same as "evidence for a lack of nonverbal rehearsal"- the latter being impossible to obtain. It is admittedly difficult to disprove the existence of nonverbal rehearsal in the absence of a good theory of what nonverbal rehearsal might look like. Clearly, visuospatial memory can be scanned covertly (see, e.g., Kosslyn, 1980). We have assumed that nonverbal rehearsal would similarly require attention and would utilize covert, spatial imagery scanning that refreshes the image (drawing in the visuospatial sketchpad?) in a way analogous to the role of subvocal articulation in verbal rehearsal. These assumptions may prove to be wrong. Nonetheless, confidence in the present conclusion is buoyed when the disconfirming evidence is drawn from converging sources, varied paradigms, multiple species, numerous distinct findings, and ample statistical power, as in this series of experiments. It is noteworthy that each experiment did result in statistically significant effects; however, in the critical comparisons between conditions that permitted versus prevented rehearsal, no reliable performance differences were observed. Finally, procedures that typically support the existence of verbal rehearsal failed here to provide evidence for nonverbal maintenance rehearsal in the present experiments. Cook, Wright, and Sands (1991) used a similarly potent paradigm (manipulating presentation rate) and convincingly reported no evidence for rehearsal of visual information by monkeys.

Of course, humans and monkeys may have the capacity for maintenance rehearsal in visual memory, but fail routinely to do so. One might argue, for example, that subjects rehearse only when target stimuli are very similar to nontargets. In the present experiments, however, stimuli were randomly constructed and randomly selected on each trial, rendering it impossible for monkeys or humans to know in advance how similar the stimuli would be and whether they should rehearse. It is uncertain to what advantage an 
organism would ignore the capacity to maintain information in memory, particularly when errors are frequent as in these experiments. Nonetheless, it is possible that rehearsal in the sketchpad is too effortful, too time intensive, or simply unnecessary for most retention tasks. In such a case, researchers must either devise new tasks that will tap the capacity to rehearse visually or train/convince subjects to utilize this ability. At a minimum, the present findings suggest what nonverbal rehearsal (if it exists) does not mean.

Without a doubt, it would be fruitful to obtain clear evidence for the existence of a nonverbal rehearsal process. Investigations of the characteristics of such a process (the original goal for the present project) might generate many interesting and important findings, perhaps providing insight into the very nature of thought without language by humans and nonhuman animals. However, theoretical constructs that lack empirical support - like maintenance rehearsal for nonverbal information-impede the understanding of the processes that do provide for visuospatial memory. Despite their appeal, such constructs might best be forgotten.

\section{REFERENCES}

BADDELEY, A. D. (1986). Human memory: Theory and practice. Boston: Allyn \& Bacon.

Basden, B. H., Basden, D. R., \& Gargano, G. J. (1993). Directed forgetting in implicit and explicit memory tests: A comparison of methods. Journal of Experimental Psychology: Learning, Memory, \& Cognition, 19, 603-616.

BJoRK, R. A. (1989). Retrieval inhibition as an adaptive mechanism in human memory. In H. L. Roediger III \& F. I. M. Craik (Eds.), Varieties of memory and consciousness: Essays in honour of Endel Tulving (pp. 309-330). Hillsdale, NJ: Erlbaum.

Brandimonte, M. A., Hitch, G. J., \& Bishop, D. V. M. (1992). Verbal recoding of visual stimuli impairs mental image transformations Memory \& Cognition, 20, 449-455.

Brown, J. (1958). Some tests of the decay theory of immediate memory. Quarterly Journal of Experimental Psychology, 10, 12-21.

CoOK, R. G., WRIGHT, A. A., \& SANDS, S. F. (1991). Interstimulus interval and viewing time effects in monkey list memory. Animal Learning \& Behavior, 19, 153-163.

GRANT, D. S. (1981). Stimulus control of information processing in pigeon short-term memory. Learning \& Motivation, 12, 19-39.

Hopkins, W. D., Washburn, D. A., \& Rumbaugh, D. M. (1990). Processing of forms stimuli presented unilaterally in humans, chimpanzees (Pan troglodytes), and monkeys (Macaca mulatta). Behavioral Neuroscience, 104, 577-584.

KENDRICK, D. F., \& Rilling, M. (1984). The role of interpolated stimuli in the retroactive interference of pigeon short-term memory. Animal Learning \& Behavior, 12, 391-401.

Kendrick, D. F., Rilling, M., \& Stonebraker, T. B. (1981). Stimulus control of delayed matching in pigeons: Directed forgetting. Journal of the Experimental Analysis of Behavior, 36, 241-251.

Kosslyn, S. M. (1980). Image and mind. Cambridge, MA: Harvard University Press.

Lejeune, M., Decker, C., \& Sanchez, X. (1994). Mental rehearsal in table tennis performance. Perceptual \& Motor Skills, 79, 627-641.

MACPHAIL, E. M. (1986). Animal memory: Past, present and future. Quarterly Journal of Experimental Psychology, 38B, 349-364.

MAKI, W. S. (1981). Directed forgetting in pigeons. In N. E. Spear \& R. R.
Miller (Eds.), Information processing in animals: Memory mechanisms. Hillsdale, NJ: Erlbaum.

MORRIS, N. (1989). Spatial monitoring in visual working memory. British Journal of Psychology, 80, 333-349.

PaIvio, A. (1971). Imagery and verbal processes. New York: Holt, Rinehart \& Winston.

Peterson, L. R., \& Peterson, M. J. (1959). Short-term retention of individual verbal items. Journal of Experimental Psychology, 58, 193-198.

Phillips, W. A. (1983). Short-term visual memory. Philosophical Transactions of the Royal Society of London: Series B, 302, 295-309.

Phillips, W. A., \& BadDELEY, A. D. (1971). Reaction time and shortterm visual memory. Psychonomic Science, 22, 73-74.

Phillips, W. A., \& Christie, D. F. M. (1977a). Components of visual memory. Quarterly Journal of Experimental Psychology, 29, 117-133.

Phil.lips, W. A., \& CHRISTIE, D. F. M. (1977b). Interference with visualization. Quarterly Journal of Experimental Psychology, 29, 637-650.

PosNer, M. I., BoIES, S. J., EICHELMAN, W. H., \& TAYLOR, R. L. (1969). Retention of visual and name codes of single letters. Journal of Experimental Psychology, 79, 1-16.

Rilling, M. E., \& NEIWORTH, J. J. (1986). Comparative cognition: A general process approach. In D. F. Kendrick, M. E. Rilling, \& M. R. Denny (Eds.), Theories of animal memory (pp. 19-33). Hillsdale, NJ: Erlbaum.

RoITBLAT, H. L. (1987). Introduction to comparative cognition. New York: Freeman.

Rumbaugh, D. M., Richardson, W. K., Washburn, D. A., SavageRumbaugh, E. S., \& Hopkins, W. D. (1989). Rhesus monkeys ( $M a-$ caca mulatta), video tasks, and implications for stimulus-response spatial contiguity. Journal of Comparative Psychology, 103, 32-38.

Sands, S. F., Urcuioli, P. J., Wright, A. A., \& Santiago, H. C. (1984). Serial position effects and rehearsal in primate visual memory. In $H$. L. Roitbalt, T. G. Bever, \& H. S. Terrace (Eds.), Animal cognition (pp. 375388). Hillsdale, NJ: Erlbaum.

SANDS, S. F., \& WRIGHT, A. A. (1980). Serial probe recognition performance by a rhesus monkey and a human with 10 - and 20 -item lists. Journal of Experimental Psychology: Animal Behavior Processes, 6 , 386-396.

ToMs, M., Morris, N., \& Foley, P. (1994), Characteristics of visual interference with visual working memory. British Journal of Psychology, 85, 131-144.

WAGNER, A. R. (1978). Expectancies and the priming of STM. In S. H. Hulse, H. Fowler, \& W. K. Honig (Eds.), Cognitive processes in animal behavior (pp. 177-220). Hillsdale, NJ: Erlbaum.

WASHBURN, D. A. (1994). Stroop-like effects for monkeys and humans: Processing speed or strength of association? Psychological Science, 5 , 375-379.

WASHBuRn, D. A., \& GulLedge, J. P. (1995). Game-like tasks for comparative research: Leveling the playing field. Behavior Research Methods, Instruments, \& Computers, 27, 235-238.

Washburn, D. A., \& Rumbaugh, D. M. (1991). Ordinal judgments of Arabic symbols by macaques (Macaca mulatta). Psychological Science, 2, 190-193.

Washrurn, D. A., \& Rumbaugh, D. M. (1994). Training rhesus monkeys (Macaca mulatta) using the Computerized Test System. In J. R. Anderson, J. J. Roeder, B. Thierry, \& N. Herrenschmidt (Eds.), Current primatology: Vol. III. Behavioural neuroscience, physiology and reproduction (pp. 77-83). Strasbourg: Université Louis Pasteur.

WIXTED, J. T. (1989). Nonhuman short-term memory: A quantitative reanalysis of selected findings. Journal of the Experimental Analysis of Behavior, 52, 409-426.

ZERVAS, Y., \& KAKKOS, V. (1991). Visuomotor behavior rehearsal in archery shooting performance. Perceptual \& Motor Skills, 73, 1183-1190.

(Manuscript received July 12, 1996; revision accepted for publication January 22, 1997.) 